\title{
The ubiquitin ligase CHIP inactivates NF-кB signaling and impairs the ability of migration and invasion in gastric cancer cells
}

\author{
FEI LIU ${ }^{1,2}$, JUN ZHOU ${ }^{1}$, PENG ZHOU ${ }^{1}$, WEICHANG CHEN ${ }^{2}$ and FENG GUO ${ }^{1}$ \\ ${ }^{1}$ Central Laboratory, ${ }^{2}$ Department of Gastroenterology, The First Affiliated Hospital \\ of Soochow University, Suzhou 215006, P.R. China
}

Received October 22, 2014; Accepted December 17, 2014

DOI: 10.3892/ijo.2015.2893

\begin{abstract}
Ubiquitin modification of proteins influences cellular processes related to carcinogenesis. The carboxyl terminus of Hsc-70-interacting protein (CHIP), as U-box-type ubiquitin ligase, induces ubiquitination and proteasomemediated degradation of its substrate proteins. In this study, the role of CHIP in diverse aspects of gastric cancer cells was investigated. CHIP overexpression in the AGS gastric cancer cells caused impaired tumor growth. CHIP overexpression significantly inhibited the migration and invasion of the AGS cells. Moreover, we found that not only RelA/p65 but also RelB, the NF- $\kappa \mathrm{B}$ subunits, was negatively regulated by CHIP, likely owing to the TRAF2 reduction. Downregulated target genes of NF- $\kappa \mathrm{B}$ subunits, including MMP-2 and -9, integrin $\beta-1$ and $\mathrm{Bcl}-2$ were involved in these processes. We also showed that the expression level of CHIP was frequently decreased in gastric cancer tissues and the low level of CHIP expression might be an indicator of an unfavorable prognosis. Taken together, these observations provide functional evidence for CHIP behaviors as a tumor suppressor in gastric cancer.
\end{abstract}

\section{Introduction}

Although global statistics show that gastric cancer is the fourth most common cancer in men and the fifth most common cancer in women, the mortality from gastric cancer ranks third to lung and liver cancer (1). Advances in diagnosis and therapeutics have improved long-term survival for early gastric cancer; however, the prognosis of advanced and metastatic

Correspondence to: Dr Feng Guo, Central Laboratory, The First Affiliated Hospital of Soochow University, Shizi Road 188, Suzhou 215006, P.R. China

E-mail: guofeng27@suda.edu.cn

Dr Weichang Chen, Department of Hematology, The First Affiliated Hospital of Soochow University, Shizi Road 188, Suzhou 215006, P.R. China

E-mail: weichangchen@126.com

Key words: CHIP, migration, invasion, NF-кB, TRAF2 gastric cancer is still not satisfactory. A better understanding of the molecular pathologies of metastatic gastric cancer will lead to progress in both diagnostics and treatment.

Metastasis is a critical determinant of cancer morbidity. Tumor cell metastasis is a multiphasic process including cell transformation, growth, angiogenesis, invasion, dissemination and survival in the circulation and cell growth at distant sites from the primary tumor location. Each step requires distinct molecular program regulating the interaction between the tumor cells, the extracellular matrix (ECM) and the cells of the stroma. Molecules participating in these steps can also be considered as potential prognostic factors. Diverse molecular regulators, including adhesion receptor families, receptor tyrosine kinase, cytoskeleton protein, adapters and signaling molecules, are involved in the regulation of migration and invasion to tumor cells (2).

The carboxyl terminus of Hsc-70-interacting protein (CHIP), encoded by the STUB1 gene, was first cloned and characterized in human heart (3). CHIP contains two important domains. The N-terminal domain of CHIP consists of three tetratricopeptide repeat (TPR) domains mediating proteinprotein interactions, and a U-box domain at its C-terminus displaying E3 ubiquitin ligase activity (4). CHIP functions in maintaining the protein homeostasis in the cytoplasm by promoting the ubiquitination and depredation of chaperonebound and misfolded proteins including glucocorticoid receptor (GR), cystic fibrosis transmembrane conductance regulator (CFTR) and mutated superoxide dismutase 1 (SOD1) (5-7). CHIP is also involved in the degradation of multiple oncogenic proteins.

Though the exact function of CHIP in tumorigenesis has not been uncovered, accumulated evidence indicates that CHIP predominantly functions as a tumor suppressor. Several studies indicate that CHIP expression can be considered as an independent prognostic factor for cancer patients including gastric, breast and colorectal cancers (8-10). In human colorectal cancer cells, CHIP inhibits the malignancy possibly through negatively regulating the $\mathrm{NF}-\kappa \mathrm{B}$ activity (10). In breast cancer cells, CHIP is implicated in the modulation the stability of estrogen receptor (ESRI) and Her-2/neu (ERBB2). CHIP silencing in breast cancer cells results in rapid tumor growth and other malignant phenotypes (11). CHIP overexpression in the MDA-MB-231 breast cancer cells promotes the ubiquitination of TNF receptor-associated factor 2 (TRAF2) 
and subsequently inactivates the TRAF2-NF- $\kappa$ B signaling (12). In gastric cancer cells, it has been reported that CHIP can degrade the classical NF- $\mathrm{BB}$ member RelA/p65 and inhibit the IL-8-induced angiogenesis (13).

However, the role of CHIP in the metastasis of gastric cancer is still poorly understood. In this study, we investigated the functional importance and potential mechanism of CHIP in the migration and invasion in gastric cancer.

\section{Materials and methods}

Clinical samples. Gastric cancer tissues were obtained, with written consent, from 164 gastric cancer patients at the Department of General Surgery, the First Affiliated Hospital of Soochow University, from 2008 to 2013. All patients had an accurate pathology diagnosis and underwent surgery without chemotherapy or radiotherapy before operation.

Tissue culture. The human gastric cancer cell line AGS was purchased from American Type Culture Collection (ATCC). All cell lines were cultured in F-12 (Hyclone, HAM'S/F-12) supplemented with $10 \%$ fetal bovine serum (FBS), $100 \mathrm{U} / \mathrm{ml}$ penicillin, $100 \mu \mathrm{g} / \mathrm{ml}$ streptomycin and $2 \mathrm{mM}$ glutamine. The cells were cultured at $37^{\circ} \mathrm{C}$ in a humidified atmosphere of $5 \% \mathrm{CO}_{2}$.

Transfection. CHIP overexpression vector was constructed based on the MSCV vector containing GFP. Full-length CDS of CHIP was amplified by PCR from human breast tissue and cloned into MSCV-GFP vector (MSCV-GFP-hCHIP). Primer sequences of CHIP are as follows: 5'-AAAAAGATCTG GCGGCATGAAGGGCAAGG-3' and 5'-GGGAACCTCAGT AGTCCTCCACCC-3'. Phoenix A packaging cells were transfected with MSCV-GFP-hCHIP or control vector (MSCV-GFP) by FuGENE HD (Roche, China). Lentivirus supernatants were used to infect the target cells.

Quantitative real-time PCR ( $q R T$-PCR). Equivalent amounts of RNA ( $2 \mu \mathrm{g})$ were reverse-transcribed with Superscript M-MLV (Promega, China). Triplicates were performed for all qRT-PCR reactions with a LightCycler 480 System (Roche). Primers for qRT-PCR were designed using Primer-BLAST (PubMed). Primers were synthesized from Invitrogen (China). The housekeeping gene $(\beta$-actin) and target genes were reverse transcribed together in a single run. The reaction with $2 \mathrm{X}$ LC480 SYBR Green I Master Mix (Roche) was according to the manufacturer's protocol. For data analysis, a target gene transcript was quantified in comparison to the house keeping gene ( $\beta$-actin) as a reference.

Western blot analysis. Whole-cell extracts were prepared with RIPA buffer according to standard procedures. Proteins were fractionated on an SDS-PAGE gel and transferred to PVDF membranes. After blocking, membranes were probed with different antibodies (Abs). Membranes were then washed and incubated with an appropriate secondary antibody. Proteins were detected and scanned with the Gel Imaging system (PeiQing, China). Band density was normalized to the $\alpha$-tubulin, actin or GAPDH reference. Abs against Bcl-2 (sc-7382), Cyclin D1 (sc-20044), TRAF-2 (sc-876), RelA (sc-372), p50 (sc-7178), p100/p52 (sc-3017),
RelB (sc-226) and c-Rel (sc-70) were purchased from Santa Cruz Biotechnology. Abs against Bim (\#2819), AKT (\#4691), p-AKT (Ser473) (\#4060), p-AKT (Thr308) (\#2965), TRAF-3 (\#4729), ITGB1 (\#9699), MMP-2 (\#4022), MMP-9 (\#2270) and CHIP (\#2080) were obtained from Cell Signaling Technology. $\alpha$-tubulin (AJ1034a), actin (AT0001) and GAPDH (\#5174) were purchased from Abgent.

Cell growth assay. Cell growth was performed with the xCelligence RTCA instrument (Roche). In this assay, impedance for indicated times was continuously monitored by the system and the value was indicated as 'Cell index' which was determined by the number of cells seeded, the overall size and morphology of the cells and the degree to which the cells interact with the sensor surface. Cell Index was continuously monitored by the system and data were collected and analyzed by RTCA software 1.2.

Cell cycle analysis. The cells were detected using flow cytometry. After 48-h continuous culture, cells were harvested and fixed by $70 \%$ ethanol for $24 \mathrm{~h}$ at $4^{\circ} \mathrm{C}$. Then single cell suspensions were prepared to stain DNA using PI staining based on the manufacturer's instructions. Cell cycle was measured by FACSCalibur (BD Biosciences, China) with at least three independent experiments performed.

Ki-67 assay. Cells were cultured in F-12 medium supplemented with $10 \%$ fetal bovine serum on cover slides. After continuous culture for 24,48 and $72 \mathrm{~h}$, the slides were fixed in cold $4 \%$ paraformaldehyde for $30 \mathrm{~min}$ and treated with $1 \%$ Triton PBS solution for $10 \mathrm{~min}$. Then 1X PBS with $10 \%$ FBS was used to block the cells for $45 \mathrm{~min}$ at room temperature. Cells were incubated with a Ki-67 antibody (1:100, Santa Cruz Biotechnology, USA). Following 1X PBS washing, sections were incubated for $30 \mathrm{~min}$ using the secondary antibody (rabbit anti-mouse IgA-B, GK500705, GTVision III). Finally, 3,3-diaminobenzine (DAB) was used to visualize the immunoreactive products. Results were evaluated by the System Microscope IX71 (Olympus, Japan).

Terminal nucleotidyl transferase-mediated nick end labeling (TUNEL) assay. Cells were cultured on cover slides for 24, 48 and $72 \mathrm{~h}$ in a humidified incubator at $37^{\circ} \mathrm{C}$ and $5 \% \mathrm{CO}_{2}$. According to the manufacturer's instructions of TUNEL system kit (Roche), the slides were fixed by cold $4 \%$ paraformaldehyde for $30 \mathrm{~min}$. Following $1 \mathrm{X}$ PBS washing, $3 \% \mathrm{H}_{2} \mathrm{O}_{2}$ methanol solution was used to block the slides for $10 \mathrm{~min}$ at $20^{\circ} \mathrm{C}$. Then the slides were treated using $1 \%$ Triton PBS solution for $2 \mathrm{~min}$ on ice after PBS washing. Avoiding light, $50 \mu \mathrm{l}$ of TUNEL reaction solution was applied to incubate the cells on slides for $60 \mathrm{~min}$ at $37^{\circ} \mathrm{C}$. Following PBS washing, the signals of TUNEL were converted using peroxidase (POD) for $30 \mathrm{~min}$ at $37^{\circ} \mathrm{C}$ and the sections were treated with DAB for $3 \mathrm{~min}$ at room temperature. Results were examined by the light System Microscope IX71 (Olympus, Japan).

Cell migration assay. Cell migration was also performed with the xCelligence RTCA instrument (Roche). In this assay, CIM-plate with upper chamber and lower chamber was used. F-12 (180 $\mu \mathrm{l})$ supplemented with 10\% FBS was added in each 
wells on the lower chamber. Cells were suspended in F-12 FBS-free media, 60,000 cells/well were added in the upper chamber. After attachment, cell migration towards lower chamber containing $10 \%$ FBS media was continuously monitored by the system and data were collected and analyzed by RTCA 1.2 software.

Scratch healing assay. For the wound healing assays, cells were treated with $10 \mathrm{mg} / \mathrm{ml}$ mitomycin C (Sigma, USA) for $3 \mathrm{~h}$. Then, cells were wounded using a pipette tip, and F-12 with $10 \%$ FBS was added. The wound closure was observed for $48 \mathrm{~h}$, with the light System Microscope IX71. The wound healing ability was calculated, compared to the width of wound closure for $0 \mathrm{~h}$.

Cell invasion assay. Cell invasion was also performed with the xCelligence RTCA instrument (Roche). In this assay, CIM-plate with two chambers were used. F-12 (180 $\mu \mathrm{l})$ supplemented with $10 \%$ FBS was added to each well in the lower chamber. Cells were suspended in F-12 FBS-free media, then 60,000 cells/well were added to the uppper chamber. After attachment, cells invading through Matrigel (cat. no. 356234, BD Biosecience, China) towards lower chamber containing $10 \%$ FBS media were continuously monitored by the system and data were collected and analyzed by RTCA 1.2 software.

Gelatinase zymography. For the assessment of MMP-2 and -9, gelatin zymography assays were used. Gelatinase zymography was performed in an $8 \%$ SDS-PAGE gel in the presence of $0.1 \%$ gelatin under non-reducing conditions. Culture media with sample buffer were loaded for SDS-PAGE with Tris-glycine SDS buffer. Samples were not boiled before electrophoresis. Following electrophoresis, the gels were washed twice in $2.5 \%$ Triton $\mathrm{X}-100$ for $30 \mathrm{~min}$ at room temperature to remove SDS. The gels were then incubated at $37^{\circ} \mathrm{C}$ overnight in substrate buffer containing $50 \mathrm{mM}$ Tris- $\mathrm{HCl}$ and $10 \mathrm{mM}$ $\mathrm{CaCl}_{2}$ at $\mathrm{pH} 8.0$ and stained with $0.5 \%$ Coomassie Blue R250 in $50 \%$ methanol and $10 \%$ glacial acetic acid for $30 \mathrm{~min}$ and destained. Upon renaturation of the enzyme, the gelatinases digested the gelatin to produce clear bands against an intensely stained background.

Immunohistochemistry (IHC). Tissue IHC was performed using a standard peroxidase-based staining method. Tissue sections $(4 \mu \mathrm{m})$ were incubated in a dry oven at $60^{\circ} \mathrm{C}$ for $1 \mathrm{~h}$ and then dewaxed in xylene for $3 \times 10 \mathrm{~min}$, rehydrated with graded ethanol in 100, 100, 95, 90, 80 and $70 \%$ ethanol for 5 min each. Then antigen retrieval was performed by pretreatment of the slides in $0.01 \mathrm{M}$ citrate buffer ( $\mathrm{pH}$ 6.0) using a microwave oven. Subsequently, the sections were treated with $3 \%$ hydrogen peroxide $\left(\mathrm{H}_{2} \mathrm{O}_{2}\right)$ for $10 \mathrm{~min}$ in order to block endogenous peroxidase. The sections were washed with $1 \mathrm{X}$ phosphate buffered saline (PBS) ( $\mathrm{pH} 7.4)$ and were incubated with rabbit anti-CHIP antibody (dilution 1:200; CST) overnight at $4^{\circ} \mathrm{C}$. The sections were then washed with $1 \mathrm{X}$ PBS and incubated with biotinylated goat anti-rabbit IgG. For each sample, the omission of primary antibody was used as a negative control. Finally, 3, 3-diaminobenzine (DAB) was used to visualize the immunoreactive products. The results were evaluated by the System Microscope IX71. The staining index was expressed as the proportion of positive staining cells $(<25 \%,+; 25-50 \%,++; 50-75 \%,+++;>75 \%,++++)$.

Statistical analysis. All experiments were repeated at least three times. The data were expressed as the mean \pm standard deviation from experiments in replicate. All statistical analysis was performed by Graphpad software. The differences between groups were valued using Student's t-test. A P-value of $\leq 0.05$ was considered significant and a P-value of $\leq 0.01$ as highly significant.

\section{Results}

Establishing a CHIP overexpressing cell line. To establish a CHIP overexpressing cell line, the reconstructed plasmid carrying both green fluorescent protein (GFP) and human CHIP cDNA were transfected into the AGS human gastric cancer cells. The individual monoclones were subsequently selected by puromycin with a final concentration of $5 \mathrm{ng} / \mu 1$ for two weeks. In parallel, an AGS cell line expressing GFP only was established as a control. The selected monoclones were further expanded and examined for the CHIP expression by qRT-PCR. As shown in Fig. $1 \mathrm{~A}$, the $C H I P$ expression at mRNA level in a single clone, which was transfected with the CHIP-expressing plasmid (AGS-CHIP), was significantly higher than that in the control cells (AGS-control). The CHIP expression at protein level was also markedly increased in the AGS-CHIP cells compared to that in the AGS-control cells by western blotting (Fig. 1B). The induced expression of CHIP was further confirmed by an IHC assay (Fig. 1C). When observed under fluorescence microscopy, both the established AGS-CHIP and the AGS-control cells presented strong GFP signals (Fig. 1D). The purities of the two established cell lines were 94.37 and $95.98 \%$, respectively, which were examined by flow cytometry (Fig. 1E). This indicated that the AGS gastric cell line overexpressing the human CHIP was established successfully.

CHIP overexpression inhibits AGS cell growth. The cell growth affected by the CHIP overexpression was detected by a real-time xCelligence system using E-plates. The AGS cells overexpressing CHIP grew much slower than that of the AGS-control cells and there was a statistically significant difference between the two established cell lines during the 72-h continuous monitoring (Fig. 2A). The cell cycle analysis and cellular DNA content measurement were examined by flow cytometry and no obvious differences were observed between the AGS-hCHIP and the AGS-control cells in any of the three phases $\left(G_{0}-G_{1}, S\right.$ and $\left.G_{2}-M\right)$ (Fig. $2 B$ ). To investigate whether CHIP overexpression affects the proliferation capability of the AGS cells, a Ki-67 cell proliferation assay was performed. The frequencies of Ki-67-positive cells in the AGS-hCHIP group were $13.78 \pm 0.50,13.11 \pm 0.50$ and $12.45 \pm 0.70 \%$; while those of $\mathrm{Ki}-67$-positive cells in the AGS-control group were $80.11 \pm 0.7,78.56 \pm 1.00$ and $76.78 \pm 0.50 \%$ at 24,48 and $72 \mathrm{~h}$, respectively (Fig. $2 \mathrm{C}$ ). The TUNEL assay was carried out to quantitatively analyze the apoptotic cells. As showed in Fig. 2D, both the AGS-hCHIP and the AGS-control cells underwent apoptosis in a timedependent manner. The percentages of apoptotic cells in 
A

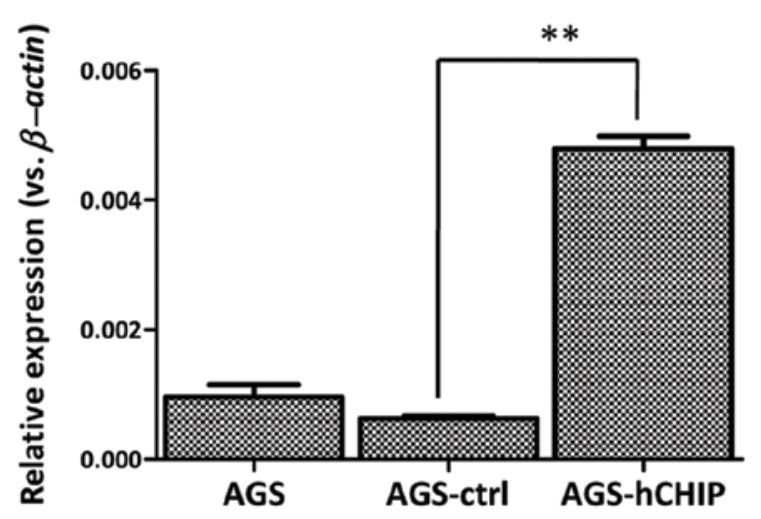

C

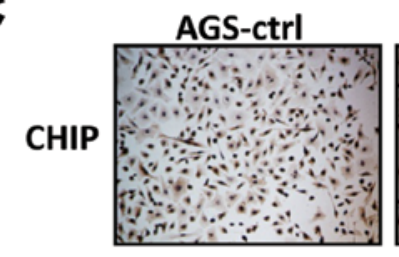

D

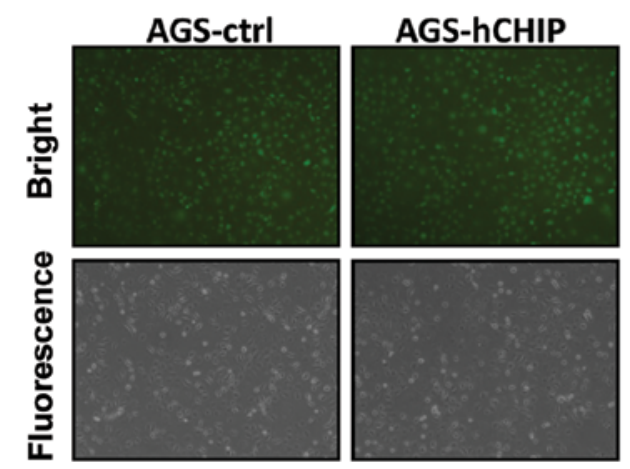

B

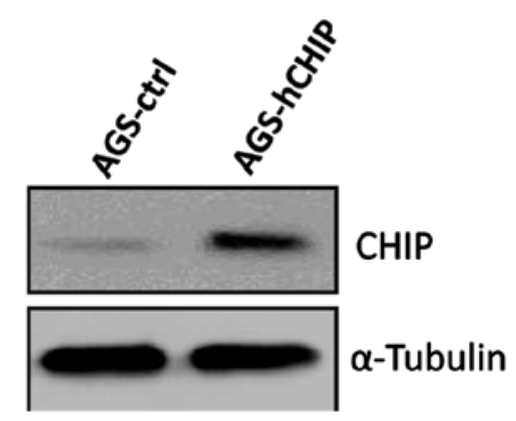

$\mathbf{E}$
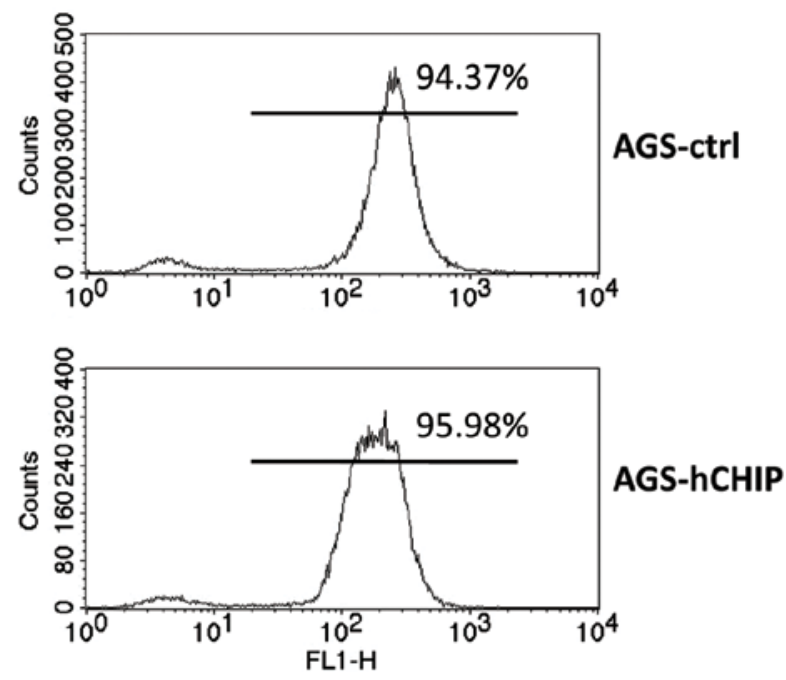

Figure 1. Establishing a CHIP-overexpressing cell line. (A) CHIP mRNA expression between the two established cell lines. $\beta$-actin normalized gene expression, measured in triplicates is displayed. Data were analyzed with Pfaffl method that provides a means for quantification of a target gene transcript in comparison to $\beta$-actin gene. Significant differences are indicated (Student's t-test, ${ }^{*} \mathrm{p}<0.05 ;{ }^{* *} \mathrm{p}<0.05$ ). (B) Protein levels of CHIP expression in the AGS-ctrl and AGS-hCHIP cell lines were determined by western blotting. The level of each protein was normalized against $\alpha$-tubulin. (C) Representative images of IHC staining using CHIP antibodies. Original magnification x40. (D) Representative images of GFP signals between the two established cell lines. GFP signals in AGS-ctrl and AGS-hCHIP cells were observed under fluorescence microscopy. The upper two images, original magnification x40, under the fluorescent field, while the lower two images showed the bright field. (E) The GFP signals of the AGS-ctrl and AGS-hCHIP cell lines were examined by flow cytometry.

the AGS-hCHIP group were $8.22 \pm 0.50,23.78 \pm 1.50$ and $59.78 \pm 3.50 \%$; while those of apoptotic cells in the AGS-control cells were $5.78 \pm 0.50,9.78 \pm 0.80$ and $23.22 \pm 1.50 \%$ at 24,48 and $72 \mathrm{~h}$, respectively. Taken together, it is shown that CHIP overexpression in AGS cells led to increased apoptosis and decreased cellular proliferation. Therefore, CHIP plays a pivotal role in cell growth of the AGS gastric cancer cells due to regulation of apoptosis and cellular proliferation.

CHIP overexpression regulates Bcl-2 expression. The mRNA expression of the pro-apoptotic genes including Bax, Bim and $\mathrm{Mcl}-1$, as well as the anti-apoptotic genes including $\mathrm{Bcl}-2$, $B c l-x l$, cIAPl and A20, were measured by qRT-PCR. As shown in Fig. 3A, the expression of the $B c l-2$ gene at the mRNA level was markedly decreased in the AGS-hCHIP cells compared to that of the AGS-control cells. The reduced Bcl-2 expression was also detected at the protein level by western blotting in the AGS-hCHIP cells (Fig. 3B). No significant changes were observed in other apoptosis-associated genes including Bax, Bim, Mcl-1, Bcl-xl, cIAP1 and A20 at mRNA level. The expression of Cyclin D1 was also comparable between the AGS-control and AGS-hCHIP cells (Fig. 3B).

The AKT signaling molecules were examined to investigate whether the AKT signaling pathway is involved in the cellular proliferation affected by the CHIP overexpression. The expression level of AKT was similar in the whole-cell extracts of the AGS-control and AGS-hCHIP cells. However, CHIP overexpression led to a clear reduction in the expression levels 
A

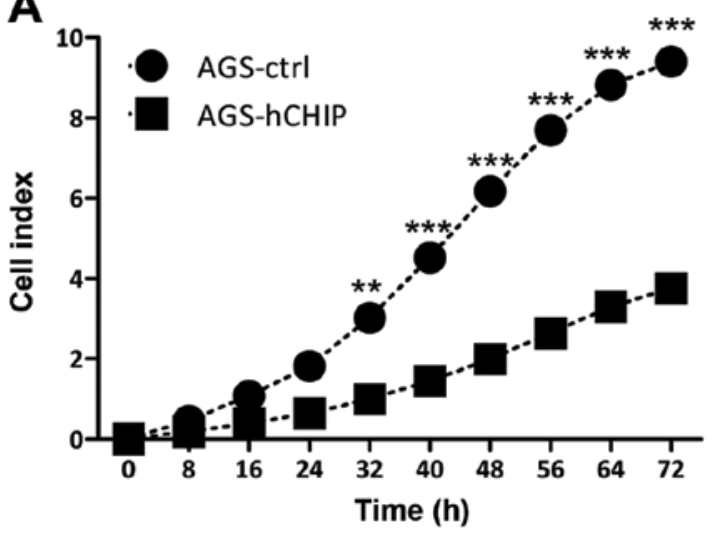

B
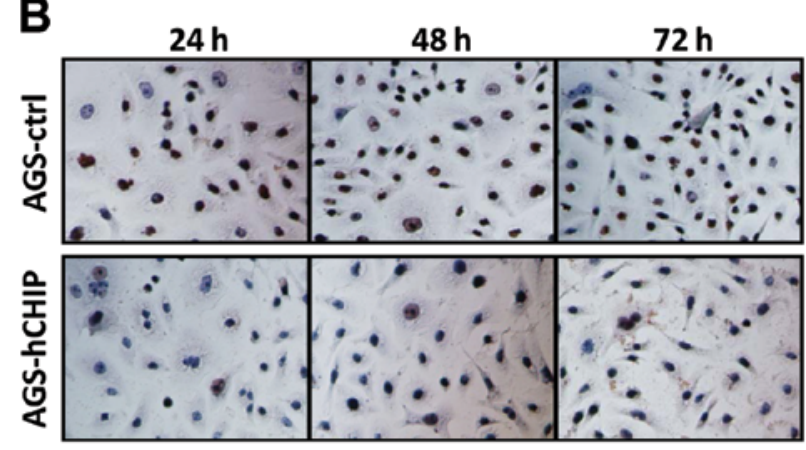

C

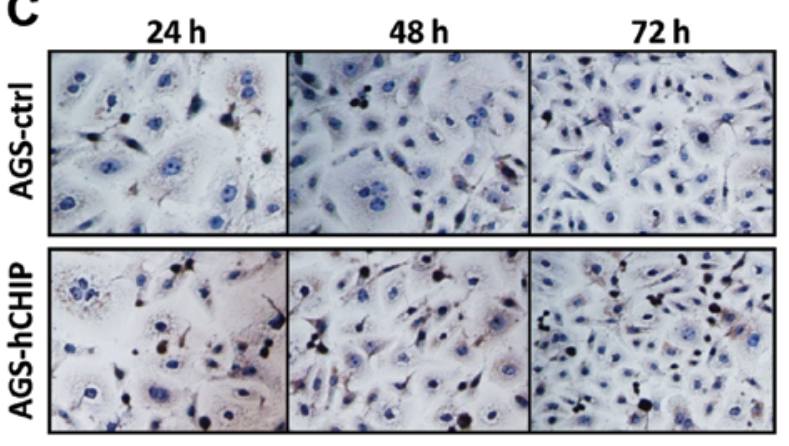

D

\begin{tabular}{c|cc}
\hline & AGS-ctrl & AGS-hCHIP \\
\hline G0-G1 (\%) & $70.07 \pm 7.22$ & $64.11 \pm 4.26$ \\
\hline S (\%) & $18.97 \pm 3.66$ & $21.36 \pm 1.78$ \\
G2-M (\%) & $11.06 \pm 3.92$ & $14.39 \pm 3.59$ \\
\hline
\end{tabular}
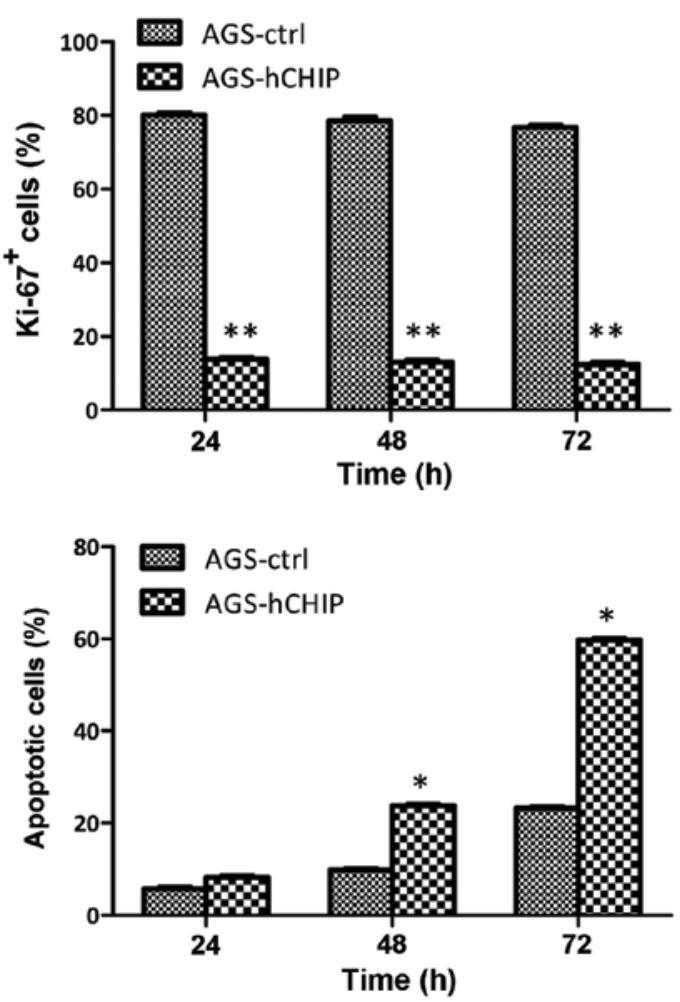

Figure 2. CHIP overexpression inhibits AGS cell growth. (A) The cell growth curves between the AGS-ctrl and AGS-hCHIP cells were detected by xCelligence system. Each plate was inoculated with 5,000 cells and the cell growth was detected during a 72-h continuous monitoring. (B) The cell cycle analysis between the two established cell lines was examined by flow cytometry. The table presents the data of three phases $\left(\mathrm{G}_{0}-\mathrm{G}_{1}, \mathrm{~S}\right.$ and $\left.\mathrm{G}_{2}-\mathrm{M}\right)$. $(\mathrm{C})$ The $\mathrm{Ki}-67$ assay was carried out to quantitatively analyze the proliferation capability. The bar chart represents the frequencies of Ki-67-positive cells of the two established cell lines. (D) The TUNEL assay was carried out to quantitatively analyze the apoptotic cells. The bar chart represents the percentage of apoptotic cells of the two established cell lines.

of p-AKT (Ser473) and p-AKT (Thr 308) (Fig. 3C). Taken together, these results indicated that the increased apoptosis in the CHIP-overexpressing AGS cells is likely attributed to the inhibition of the Bcl-2 expression. The suppressed phosphorylation of AKT due to CHIP overexpression is involved in the reduced cellular proliferation.

CHIP negatively regulates the $N F-\kappa B$ signaling. The expression of RelA/p65 and p50, representing the canonical NF- $\kappa \mathrm{B}$ activities, was clearly decreased in both the cytoplasmic (CE) and nuclear fraction (NE) in the AGS-hCHIP cells compared to that in the AGS-control cells. Moreover, the expression of RelB, representing the non-canonical NF- $\kappa \mathrm{B}$ activity, was clearly reduced in the cytoplasmic fraction but not in nuclear fraction of the AGS-hCHIP cells. However, no obvious changes in the expression of p52 and c-Rel were observed between the AGS-hCHIP cells and the AGS-control cells (Fig. 4A). TRAF2, which positively regulates both the canonical and the non-canonical NF- $\kappa \mathrm{B}$ activity, was also slightly decreased in the AGS-hCHIP cells. TRAF3, which negatively regulates the non-canonical NF- $\mathrm{B}$ activity, was not affected by the overexpression of CHIP (Fig. 4B). Taken together, it was indicated that CHIP overexpression suppressed the expression of TRAF2, which further inhibited not only the classical NF- $\kappa \mathrm{B}$ activity but also the alternative $N F-\kappa B$ activity in the AGS gastric cancer cells. 
A

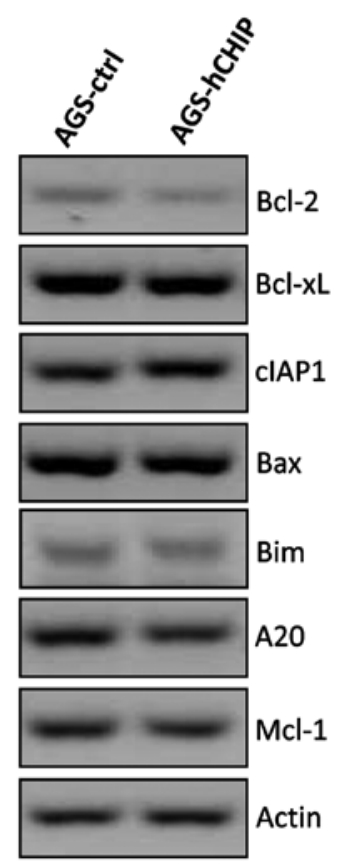

B
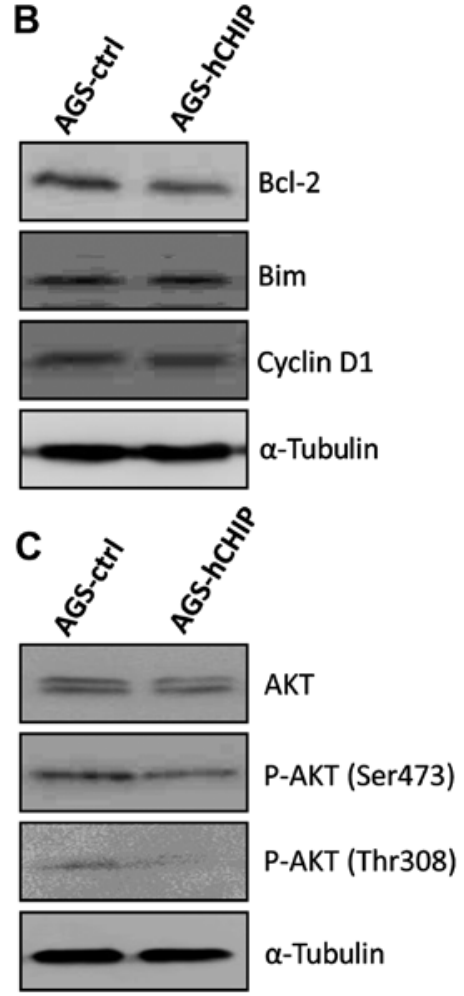

Figure 3. CHIP overexpression regulates Bcl-2 expression. (A) The mRNA expression of pro-apoptotic and anti-apoptotic genes, examined by RT-PCR (B) Bcl-2, Bim and Cyclin D1 expression at the protein level were analyzed by western blotting. The level of each protein was normalized against $\alpha$-tubulin. (C) The protein expression level of AKT, pAKT (Ser473) and pAKT (Thr308) was analyzed by western blotting. The level of each protein was normalized against $\alpha$-tubulin.

A

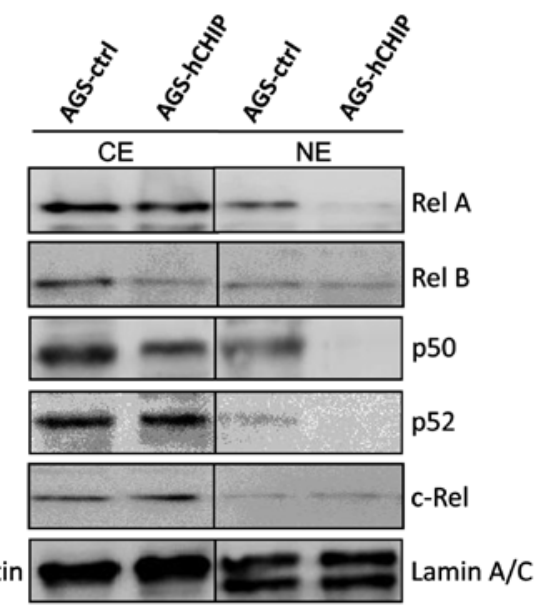

B

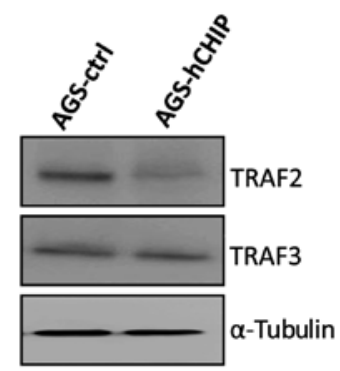

Figure 4. CHIP negatively regulates the NF- $\mathrm{BB}$ signaling. (A) The protein expression of NF- $\mathrm{KB}$ subunits were analyzed by western blotting. Protein expression in cytoplasmic (CE) and nuclear portion (NE) was normalized against actin and Lamin A/C, respectively. (B) The protein expression of TRAF-2 and TRAF-3 were analyzed by western blotting. The level of each protein was normalized against $\alpha$-tubulin.
CHIP overexpression attenuates the migration ability of AGS gastric cancer cells. The migration ability affected by the overexpression of CHIP was investigated by a real-time xCelligence system using CIM-plates. The AGS cells overexpressing CHIP migrated markedly slower than that of the AGS-control cells during the 24-h continuous monitoring and there was a statistically significantly difference in the migration assay between the two established cell lines (Fig. 5A). The in vitro scratch assay was also performed to measure quantitatively the migration ability of the cells. A scratched cell monolayer was created in both cell lines and the images were captured at the beginning and after $48 \mathrm{~h}$. At $48 \mathrm{~h}$ it was shown that the AGS-hCHIP cells migrated from the edge towards the scratch center much slower than that of the AGS-control cells (Fig. 5B). Thus, the overexpression of CHIP clearly inhibited the migration ability of the AGS cells.

CHIP overexpression affects the invasion ability of AGS gastric cancer cells. The invasion ability affected by the overexpression of CHIP was also measured by a real-time xCelligence system using Matrigel (dilution at 1:40)-coated CIM-plates. The AGS cells overexpressing CHIP invaded through Matrigel markedly slower than that of the AGS-control cells and there was a statistically significant difference in the invasion ability between the established cell lines during the 24-h continuous monitoring (Fig. 6A). The integrin $\beta-1$ expression at the protein level was also dramatically decreased in the AGS-hCHIP cells compared to that of the AGS-control cells (Fig. 6B). The gelatin zymography experiment was further performed to explore the 
A

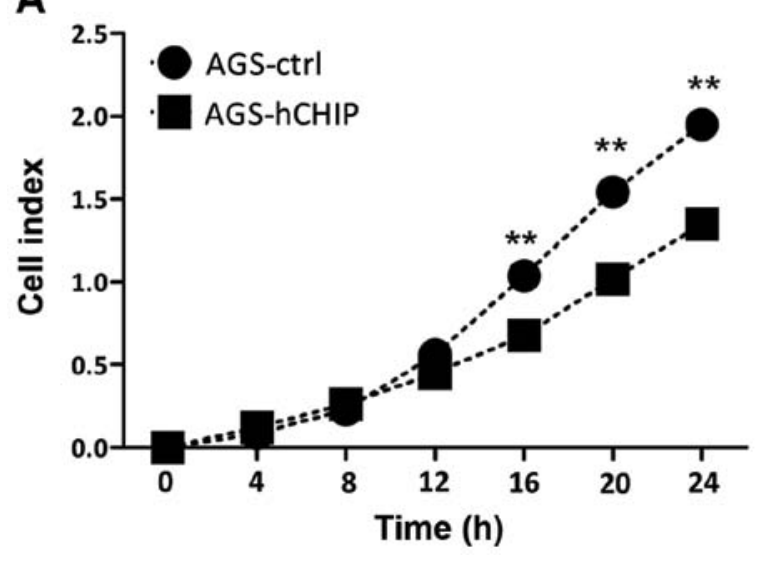

B

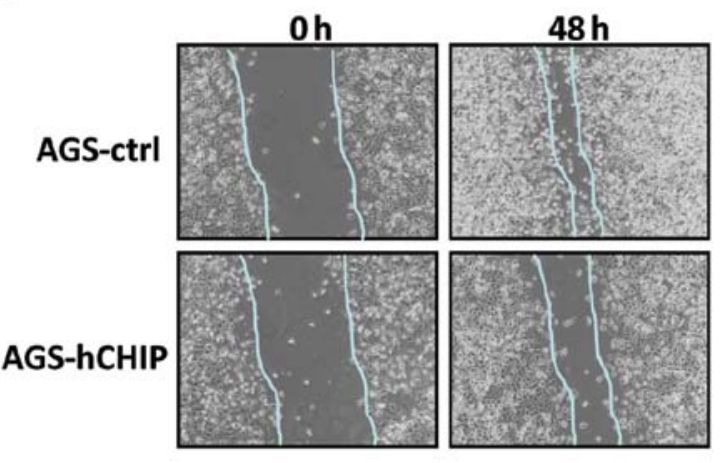

Figure 5. CHIP overexpression attenuates the migration ability of AGS gastric cancer cells. (A) The migration ability of AGS-ctrl cells and AGS-hCHIP cells was detected by a real-time xCelligence system using CIM-plates. Each plate was inoculated with 60,000 cells and the migration was detected via 24-h monitoring. (B) The migration ability of the cells was detected by the scratch healing assay at $48 \mathrm{~h}$. The blue lines represent the migration boundaries.

A

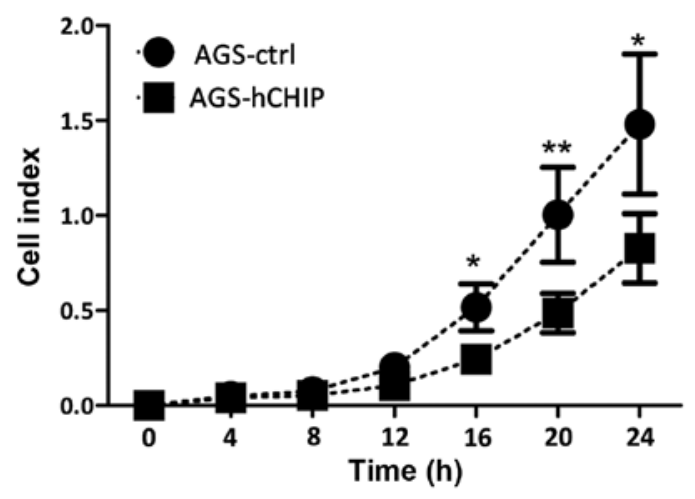

C

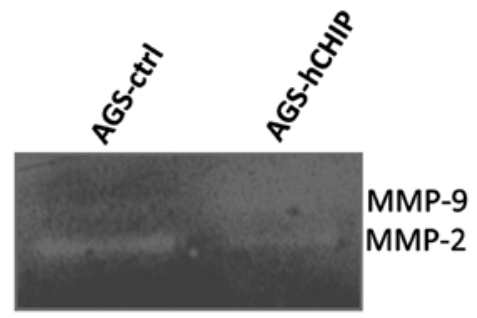

B

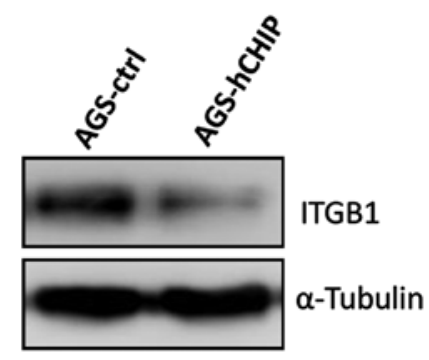

D

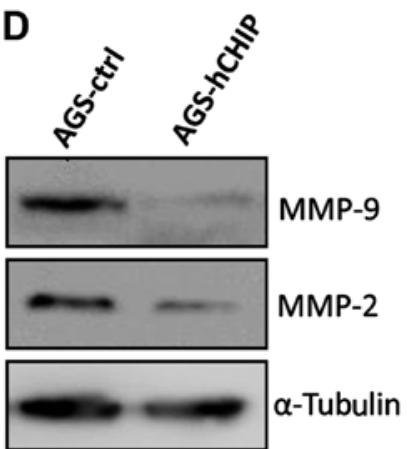

Figure 6. CHIP overexpression affects the invasion ability of AGS gastric cancer cells. (A) The invasion ability of the two established cell lines was detected by a real-time xCelligence system using Matrigel (1:40 dilution)-coated CIM-plates. Each plate was inoculated with 60,000 cells and the invasion ability was detected via 24-h monitoring. (B) The protein level of integrin $\beta-1$ was analyzed by western blotting in the two established cell lines. The level of each protein was normalized against $\alpha$-tubulin. (C) The activity of MMP-2 and -9 was detected by gelatin zymography experiments. (D) The protein level of MMP-2 and -9 was analyzed by western blotting in the two established cell lines. The level of each protein was normalized against $\alpha$-tubulin.

relative amounts of active and inactive gelatinase (MMP-2 or MMP-9). As showed in Fig. 5B, both the MMP-2 and -9 activities were decreased in the AGS-hCHIP cells compared to the AGS-control cells (Fig. 6C). Moreover, the expression of MMP-2 and -9 in the AGS-hCHIP cells at protein level was also significantly lower than the AGS-control cells (Fig. 6D). Thus, the results here indicated that overexpression of CHIP suppressed the migration and invasion ability of the AGS gastric cancer cells, which was correlated with the decreased activity of MMP-2 and -9 and the downregulated integrin $\beta-1$ expression.

CHIP expression in human gastric cancer tissues. The expression of CHIP was examined in individual human gastric cancer tissues. As shown in Fig. 7A, the average mRNA level of the CHIP gene detected in 18 gastric cancer tissues was clearly lower than that of the paired normal gastric mucosa by qRT-PCR. The western blotting was performed to examine 
A

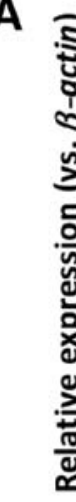

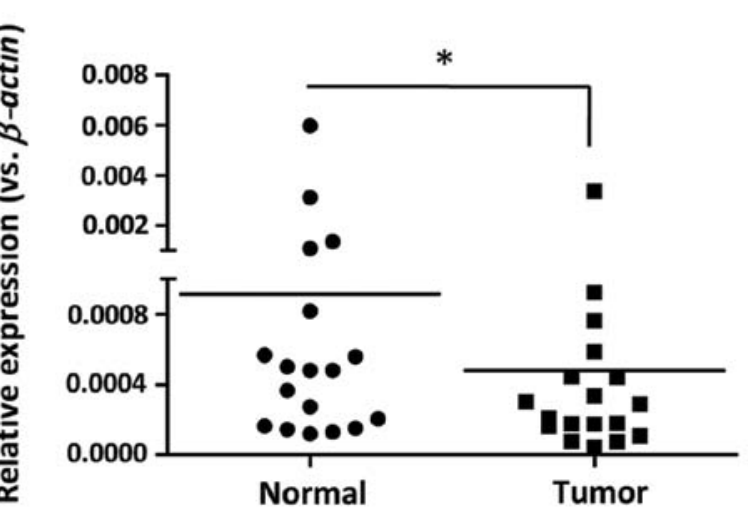

C

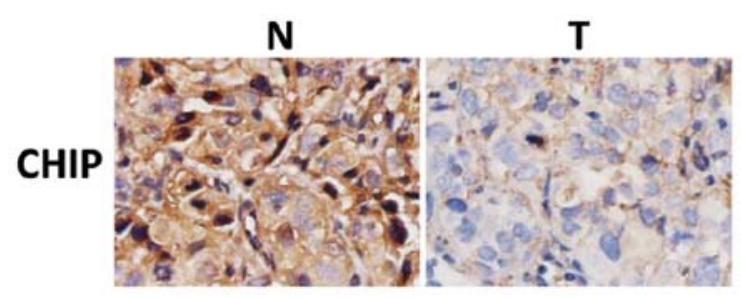

B
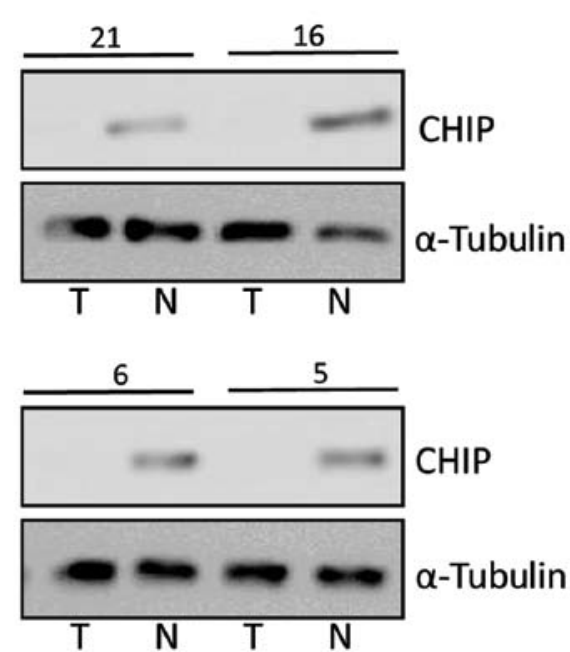

Figure 7. CHIP expression in human gastric cancer tissues. (A) CHIP mRNA expression in gastric cancers. Total RNA was prepared from normal (N) or tumor (T) tissues and the CHIP mRNA level was quantified using qRT-PCR. $\beta$-actin normalized gene expression, measured in triplicate is displayed. (B) CHIP protein level in gastric cancer tissues was analyzed by western blotting. The level of each protein was normalized against $\alpha$-tubulin. (C) Representative images of IHC staining using CHIP antibodies. Normal (N) and tumor (T) images, original magnification x40.

Table I. Association of CHIP expression with clinicopathologic features of 164 gastric cancers patients.

\begin{tabular}{lcccc}
\hline Characteristics & $\begin{array}{c}\text { No. of } \\
\text { patients } \\
\text { (total: 164) }\end{array}$ & $\begin{array}{c}\text { CHIP } \\
\text { expression } \\
+-++\end{array}$ & $\begin{array}{c}\text { CHIP } \\
\text { expression } \\
+++-++++\end{array}$ & P-value \\
& & &
\end{tabular}

\begin{tabular}{lrrrr}
\hline $\begin{array}{l}\text { Age (years) } \\
\leq 60\end{array}$ & 45 & 22 & 23 & 0.834 \\
$>60$ & 119 & 56 & 63 & \\
Gender & & & & \\
Male & 66 & 30 & 36 & 0.279 \\
Female & 88 & 33 & 55 & \\
Tumor diameter & & & & \\
(cm) & & & & \\
$\leq 5$ & 87 & 36 & 51 & 0.306 \\
$>5$ & 77 & 38 & 39 & \\
Differentiation & & & & \\
Well-moderately & 96 & 78 & 18 & $<0.001$ \\
Poorly & 68 & 6 & 62 & \\
TNM stage & & & & \\
I-II & 70 & 23 & 47 & 0.002 \\
III-IV & 84 & 49 & 35 & \\
\hline
\end{tabular}

the expression of CHIP in gastric tumor tissues at the protein level. Representative results are presented in Fig. 7B, showing that the expression of CHIP was detected in the normal tissues while the expression of CHIP was decreased in most of the gastric cancer samples or was hardly detected. The expression of CHIP was detected in both the nucleus and the cytoplasm of the normal tissues by IHC assay, while the expression of CHIP was predominantly localized in cytoplasm of the gastric tumor cells. Moreover, a strong staining of CHIP was detected in the gastric normal mucosa, while a relatively weak staining of CHIP was found in the gastric tumor cells (Fig. 7C). A total of 164 gastric cancer samples were assayed for the CHIP expression by IHC and the correlation between the expression of CHIP and the clinical features was investigated. As shown in Table I, the expression level of CHIP in gastric cancer tissue was negatively correlated with the differentiation status and the TNM stages of the gastric cancer patients, but was not correlated with age, gender and tumor diameters. These results indicated that CHIP expression was frequently decreased in gastric cancer tissues and the decreased expression of CHIP was an indicator of an unfavorable prognosis.

\section{Discussion}

In this study, we show that CHIP overexpression clearly affected the ability of migration and invasion of the AGS gastric cancer cells, which was associated with the decreased activities of MMP-2 and -9 and the downregulated integrin $\beta-1$. Not only the canonical NF- $\mathrm{B}$ but also the noncanonical NF- $\kappa \mathrm{B}$ activity was influenced by the presence of CHIP overexpression. TRAF2, which positively regulates both $\mathrm{NF}-\kappa \mathrm{B}$ signaling, was also clearly reduced. In addition, we show that CHIP played a critical role in cell growth of the AGS gastric cancer cells via regulating cellular proliferation and survival. 
In the CHIP-overexpressing AGS gastric cancer cells, the reduction of TRAF2 as well as NF- $\mathrm{KB}$ subunits was observed. A previous report suggested that CHIP affects NF- $\mathrm{KB}$ mediated cell invasion via regulating TRAF2 expression in the breast cancer cells (12). Particularly, RelA/p65, representing the classical NF- $\kappa \mathrm{B}$ activity was found downregulated in that report. In mammals, the transcription factor family of NF- $\mathrm{KB}$

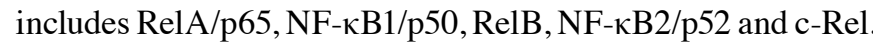
$\mathrm{NF}-\kappa \mathrm{B}$ subunits play critical roles in cell survival, inflammation, proliferation, apoptosis and tumorigenesis (14). The activation of NF- $\mathrm{KB}$ subunits triggers the canonical signaling pathway which is characterized by the activation of RelA-p50 heterodimers and the non-canonical signaling pathway that activates RelB-p52 heterodimers (15). The non-canonical NF-kB-triggering receptors contain a TRAF-binding motif, recruiting different TRAF members, particularly TRAF2 and TRAF3 $(16,17)$. TRAF2 is important for activation of the canonical NF- $\mathrm{KB}$ pathway but also mediates their stimulatory effects on the c-Jun N-terminal kinase pathway (18). In addition, TRAF2 is involved in the degradation of the NF- $\mathrm{kB}$ inducing kinase (NIK) that triggers the non-canonical NF- $\mathrm{kB}$ signaling pathway $(19,20)$. In general, TRAF2 are considered as a positive regulator of both canonical and non-canonical NF- $\mathrm{KB}$ activation. In this study, the reduction of RelA and p50 as well as RelB was observed in the presence of CHIP overexpression. Growing attention is paid to understanding the role of RelB in the pathogenesis of diverse malignancies, such as prostate cancer, breast cancer and lymphoid malignancy. Targeting RelB has also been recommended to be a valuable strategy in overcoming radiation resistance in the prostate and breast cancer $(21,22)$. The affects of CHIP on RelB would further support the idea that CHIP functions as a tumor suppressor in gastric cancer. Other NF- $\mathrm{KB}$ subunits, such as c-Rel and p52, stayed unchanged in the CHIP-overexpressing AGS gastric cancer cells. The expression of TRAF3, a negative regulator of non-canonical NF- $\kappa \mathrm{B}$ activation, was not affected by CHIP overexpression. These results suggest that in the gastric cancer cells, CHIP negatively regulates TRAF2 expression, which subsequently inhibits both canonical and non-canonical NF- $\kappa B$ expression. The potential mechanism by which CHIP negatively regulates the expression of TRAF2 remains unclear. Ubiquitination and proteasome-mediated degradation of TRAF2 by CHIP might be one of the explanations.

Gastric cancer is a very aggressive malignant tumor due to its invasive nature and early metastatic ability. Degradation of the ECM and basement membrane barriers are essential steps in the pathogenesis of gastric cancer. Many studies have confirmed the critical roles of matrix metalloproteinases (MMPs) and their inhibitors in the growth and progression of gastric cancer. The human MMPs family consists of at least 26 proteases, which are subdivided into collagenases, gelatinases, stromelysins and matrilysins (23). MMP-2 and -9 are well-characterized gelatinases and are closely associated with cancer invasion and metastasis due to their strong proteolytic activity of ECM. While MMP-2 promotes cleavage of ECM proteins, MMP-9 modulates permeability of the vascular endothelium (24). The mRNA expression of MMP-2 and -9 in gastric cancer tissue is significantly higher than that in normal tissue (25-26). The expression of MMP-2 and -9 is also correlated with clinicopathological features of tumor patients. Overexpression of MMP-2 and -9 indicates worse survival of gastric cancer patients (27-29). NF- $\kappa$ B and AP-1 are important transcription factors in regulating the activity of MMP-2 and -9, as the gene promoter contains NF- $\mathrm{KB}$ and AP-1 binding sites (30). In gastric cancer cells, MMP-2 and -9 could be also upregulated in response to IL-1 $\beta$ stimulation, which is p38-mediated and AP-1-dependent (31). In this study, we found that CHIP overexpression in the AGS gastric cancer cells inhibited the production and the activity of MMP-2 and -9 , indicating by the western blotting and gelatinase zymography assay. The migration and invasion abilities of the AGS cells were largely affected by CHIP overexpression. The data reported here is in line with the previous results that CHIP overexpression suppresses the invasion ability and inhibits the expression of MMP-9 in breast cancer cells (12). However, the expression of urokinase plasminogen activator (uPA) was not affected by the overexpression of CHIP in the AGS cells (data not shown); unlike that in the MDA-MB-231 breast cancer cells.

In this study, we also observed that the expression of integrin $\beta-1$ was downregulated in the CHIP-overexpressing gastric cancer cells. Integrin $\beta-1$, encoded by the ITGBI gene, belongs to the family of heterodimeric transmembrane cell surface receptors that contain $18 \alpha$ and $8 \beta$ subunits. Integrin $\beta-1$, as a direct target of mir-29c, positively regulates gastric cancer cell adhesion, invasion and migration (32). Overexpression of integrin $\beta-1$ has been found in various epithelial malignancies including breast cancer and glioblastoma, during invasion, angiogenesis and metastasis. High levels of integrin $\beta-1$ expression have been detected in gastric cancer and shown to be associated with poor prognosis and recurrence in patients with gastric cancer $(33,34)$. Several studies have also shown that expression of integrin $\beta-1$ is associated with peritoneal metastasis of gastric cancer and functional blocking of integrin $\beta-1$ significantly reduces the peritoneal metastasis of gastric cancer in vivo $(35,36)$. A typical binding site for NF- $\mathrm{kB}$ is located in the promoter of the human ITGBI gene (37). In a small cell lung cancer cell line (SCLC), blocking RelB expression can prevent the increase of integrin $\beta-1$ (38). Here, we found in gastric cancer cells, that CHIP negatively regulated the integrin $\beta-1$ expression. The RelB reduction would be one of the reasons for repressed integrin $\beta-1$ expression. The inhibited expression of RelA/p65 in the presence of CHIP was involved in the downregulation of MMP-2 and -9 in the gastric cancer cells. The decreased activities of MMP-2 and -9, together with the downregulated integrin $\beta-1$ contributed to the diminished migration and invasion abilities of gastric cancer cells.

CHIP is generally considered as a tumor suppressor. However, a few studies have also suggested that CHIP may have opposing roles in certain cancers, such as gliomas and esophageal squamous cell carcinoma $(39,40)$. In this study, we found that CHIP expression level was often decreased in gastric cancer patients, either in mRNA or protein levels. We also found that the level of CHIP expression was significantly associated with the differentiation status and the TNM staging, suggesting that the decreased expression of CHIP might indicate poor prognosis. These findings are in line with previous findings $(13,41)$. 
Increased apoptosis and decreased cellular proliferation, which lead to slower cell growth, were observed in the AGS cells transfected with CHIP. Clear reduction of Bcl-2, a target gene of RelB (42), would be one of the reasons for increased apoptosis. The decreased phosphoration of AKT and NF- $\mathrm{BB}$ signaling contributed to the decreased cellular proliferation. In contrast, it is reported that in normal breast epithelial MCF10A and breast cancer MCF7 cells, CHIP activates PI3K/AKT survival factors and induce apoptosis (43).

Taken together, we found that CHIP overexpression in gastric cancer cells affected several aspects of malignant phenotypes, including cell growth, migration and invasion. The defected TRAF2-NF- $\mathrm{B}$ signaling downregulated several important molecules, including apoptosis genes, MMPs and integrin $\beta-1$, involved in these processes. Particularly, RelB, representing the non-canonical $\mathrm{NF}-\kappa \mathrm{B}$, was reduced in the presence of CHIP in the gastric cancer cells. The negative correlation of CHIP expression with clinical features of gastric cancer patients support the idea that CHIP is a tumor suppressor in gastric cancer.

\section{Acknowledgements}

This study was supported by Jiangsu Provincial Natural Science Foundation of China (F.G., grant no. BK2011306) and National Natural Science Foundation of China (F.G., grant no. 81172433 and W.C.C., grant no. 81272737).

\section{References}

1. Ferlay J, Soerjomataram II, Dikshit R, et al: Cancer incidence and mortality worldwide: sources, methods and major patterns in GLOBOCAN 2012. Int J Cancer: Sep 13, 2014 (Epub ahead of print). doi: 10.1002/ijc.29210.

2. Bozzuto G, Ruggieri P and Molinari A: Molecular aspects of tumor cell migration and invasion. Ann Ist Super Sanita 46: 66-80, 2010.

3. Ballinger CA, Connell P, Wu Y, et al: Identification of CHIP, a novel tetratricopeptide repeat-containing protein that interacts with heat shock proteins and negatively regulates chaperone functions. Mol Cell Biol 19: 4535-4545, 1999.

4. McDonough $\mathrm{H}$ and Patterson C: CHIP: a link between the chaperone and proteasome systems. Cell Stress Chaperones 8: 303-308, 2003.

5. Connell P, Ballinger CA, Jiang J, et al: The co-chaperone CHIP regulates protein triage decisions mediated by heat-shock proteins. Nat Cell Biol 3: 93-96, 2001.

6. Meacham GC, Patterson C, Zhang W, Younger JM and Cyr DM: The Hsc70 co-chaperone CHIP targets immature CFTR for proteasomal degradation. Nat Cell Biol 3: 100-105, 2001.

7. Urushitani M, Kurisu J, Tateno M, et al: CHIP promotes proteasomal degradation of familial ALS-linked mutant SOD1 by ubiquitinating Hsp/Hsc70. J Neurochem 90: 231-244, 2004.

8. Patani N, Jiang W, Newbold R and Mokbel K: Prognostic implications of carboxyl-terminus of Hsc70 interacting protein and lysyl-oxidase expression in human breast cancer. J Carcinog 9: 9, 2010.

9. Sun C, Li HL, Shi ML, Liu QH, Bai J and Zheng JN: Diverse roles of C-terminal Hsp70-interacting protein (CHIP) in tumorigenesis. J Cancer Res Clin Oncol 140: 189-197, 2014.

10. Wang Y, Ren F, Feng Y, et al: CHIP/Stub1 functions as a tumor suppressor and represses NF-kappaB-mediated signaling in colorectal cancer. Carcinogenesis 35: 983-991, 2014.

11. Kajiro M, Hirota R, Nakajima Y, et al: The ubiquitin ligase CHIP acts as an upstream regulator of oncogenic pathways. Nat Cell Biol 11: 312-319, 2009.

12. Jang KW, Lee KH, Kim SH, et al: Ubiquitin ligase CHIP induces TRAF2 proteasomal degradation and NF-kappaB inactivation to regulate breast cancer cell invasion. J Cell Biochem 112: 3612-3620, 2011.
13. Wang S, Wu X, Zhang J, et al: CHIP functions as a novel suppressor of tumour angiogenesis with prognostic significance in human gastric cancer. Gut 62: 496-508, 2013.

14. Xu J, Zhou P, Wang W, Sun A and Guo F: RelB, together with RelA, sustains cell survival and confers proteasome inhibitor sensitivity of chronic lymphocytic leukemia cells from bone marrow. J Mol Med (Berl) 92: 77-92, 2014.

15. VallabhapurapUSAnd Karin M: Regulation and function of NF-kappaB transcription factors in the immune system. Annu Rev Immunol 27: 693-733, 2009.

16. Shih VF, Tsui R, Caldwell A and Hoffmann A: A single NFkappaB system for both canonical and non-canonical signaling. Cell Res 21: 86-102, 2011.

17. Sun SC: Non-canonical NF-kappaB signaling pathway. Cell Res 21: 71-85, 2011.

18. Shu HB, Takeuchi M and Goeddel DV: The tumor necrosis factor receptor 2 signal transducers TRAF2 and c-IAP1 are components of the tumor necrosis factor receptor 1 signaling complex. Proc Natl Acad Sci USA 93: 13973-13978, 1996.

19. Karl I, Jossberger-Werner M, Schmidt N, et al: TRAF2 inhibits TRAIL- and CD95L-induced apoptosis and necroptosis. Cell Death Dis 5: e1444, 2014.

20. Hayden MS and Ghosh S: NF-kappaB, the first quarter-century: remarkable progress and outstanding questions. Genes Dev 26: 203-234, 2012.

21. Holley AK, Xu Y, St Clair DK and St Clair WH: RelB regulates manganese superoxide dismutase gene and resistance to ionizing radiation of prostate cancer cells. Ann NY Acad Sci 1201: 129-136, 2010.

22. Wang X, Belguise K, O'Neill CF, et al: RelB NF-kappaB represses estrogen receptor alpha expression via induction of the zinc finger protein Blimp1. Mol Cell Biol 29: 3832-3844, 2009.

23. Lukaszewicz-Zajac M, Mroczko B and Szmitkowski M: Gastric cancer - the role of matrix metalloproteinases in tumor progression. Clin Chim Acta 412: 1725-1730, 2011.

24. Galis ZS, Muszynski M, Sukhova GK, et al: Cytokine-stimulated human vascular smooth muscle cells synthesize a complement of enzymes required for extracellular matrix digestion. Circ Res 75: 181-189, 1994.

25. Chu D, Zhang Z, Li Y, et al: Matrix metalloproteinase-9 is associated with disease-free survival and overall survival in patients with gastric cancer. Int J Cancer 129: 887-895, 2011.

26. Zhang M, Zhu GY, Gao HY, Zhao SP and Xue Y: Expression of tissue levels of matrix metalloproteinases and tissue inhibitors of metalloproteinases in gastric adenocarcinoma. J Surg Oncol 103: 243-247, 2011.

27. Alakus H, Grass G, Hennecken JK, et al: Clinicopathological significance of MMP-2 and its specific inhibitor TIMP-2 in gastric cancer. Histol Histopathol 23: 917-923, 2008.

28. Gao ZL, Zhang C, Du GY and Lu ZJ: Clinical significance of changes in tumor markers, extracellular matrix, MMP-9 and VEGF in patients with gastric carcinoma. Hepatogastroenterology 54: 1591-1595, 2007.

29. Zhou Y, Li G, Wu J, et al: Clinicopathological significance of E-cadherin, VEGF, and MMPs in gastric cancer. Tumour Biol 31: 549-558, 2010

30. Eberhardt W, Huwiler A, Beck KF, Walpen S and Pfeilschifter J: Amplification of IL-1 beta-induced matrix metalloproteinase- 9 expression by superoxide in rat glomerular mesangial cells is mediated by increased activities of NF-kappa B and activating protein-1 and involves activation of the mitogen-activated protein kinase pathways. J Immunol 165: 5788-5797, 2000.

31. Huang Q, Lan F, Wang X, et al: IL-1beta-induced activation of p38 promotes metastasis in gastric adenocarcinoma via upregulation of AP-1/c-fos, MMP2 and MMP9. Mol Cancer 13: 18, 2014.

32. Han TS, Hur K, Xu G, et al: MicroRNA-29c mediates initiation of gastric carcinogenesis by directly targeting ITGB1. Gut: May 28, 2014 (Epub ahead of print). pii: gutjnl-2013-306640. doi: 10.1136/gutjnl-2013-306640.

33. Zhao ZS, Li L, Wang HJ and Wang YY: Expression and prognostic significance of CEACAM6, ITGB1, and CYR61 in peripheral blood of patients with gastric cancer. J Surg Oncol 104: 525-529, 2011.

34. Xu ZY, Chen JS and Shu YQ: Gene expression profile towards the prediction of patient survival of gastric cancer. Biomed Pharmacother 64: 133-139, 2010.

35. Lin MT, Chang CC, Lin BR, et al: Elevated expression of Cyr61 enhances peritoneal dissemination of gastric cancer cells through integrin alpha2beta1. J Biol Chem 282: 34594-34604, 2007. 
36. Ura H, Denno R, Hirata K, Yamaguchi $\mathrm{K}$ and Yasoshima $\mathrm{T}$ : Separate functions of alpha2beta1 and alpha3betal integrins in the metastatic process of human gastric carcinoma. Surg Today 28: 1001-1006, 1998.

37. Ahmed KM, Zhang $\mathrm{H}$ and Park CC: NF-kappaB regulates radioresistance mediated by beta1-integrin in three-dimensional culture of breast cancer cells. Cancer Res 73: 3737-3748, 2013.

38. Saito T, Sasaki CY, Rezanka LJ, Ghosh P and Longo DL: p52-independent nuclear translocation of RelB promotes LPS-induced attachment. Biochem Biophys Res Commun 391: 235-241, 2010.

39. Xu T, Zhou Q, Zhou J, et al: Carboxyl terminus of Hsp70-interacting protein (CHIP) contributes to human glioma oncogenesis. Cancer Sci 102: 959-966, 2011.
40. Wen J, Luo KJ, Hu Y, Yang H and Fu JH: Metastatic lymph node CHIP expression is a potential prognostic marker for resected esophageal squamous cell carcinoma patients. Ann Surg Oncol 20: 1668-1675, 2013.

41. Gan L, Liu DB, Lu HF, et al: Decreased expression of the carboxyl terminus of heat shock cognate 70 interacting protein in human gastric cancer and its clinical significance. Oncol Rep 28: 1392-1398, 2012

42. Wang X, Belguise K, Kersual N, et al: Oestrogen signalling inhibits invasive phenotype by repressing RelB and its target BCL2. Nat Cell Biol 9: 470-478, 2007.

43. Lv Y, Song S, Zhang K, Gao H and Ma R: CHIP regulates AKT/ FoxO/Bim signaling in MCF7 and MCF10A cells. PLoS One 8: e83312, 2013. 